\title{
STOCHASTIC DYNAMICS OF QUANTUM SPIN SYSTEMS
}

\author{
ADAM W. MAJEWSKI \\ Institute of Theoretical Physics and Astrophysics, Gdańsk University \\ Wita Stwosza 57, 80-952 Gdańsk, Poland \\ E-mail: fizwam@univ.gda.pl \\ ROBERT OLKIEWICZ \\ Institute of Theoretical Physics, University of Wroctaw \\ pl. Maksa Borna 9, 50-204 Wroctaw, Poland \\ E-mail:rolek@proton.ift.uni.wroc.pl \\ BOGUSEAW ZEGARLIŃSKI \\ Mathematics Department, Imperial College \\ 180 Queen's Gate, London, SW7 2BZ, UK \\ E-mail: b.zegarlinski@ic.ac.uk
}

\begin{abstract}
We show that recently introduced noncommutative $L_{p}$-spaces can be used to constructions of Markov semigroups for quantum systems on a lattice.

1. Introduction. The aim of this paper is to give a brief exposition of explicit constructions of some quantum dynamical semigroups of Markov type with a clear physical interpretation. To define such a semigroup let us consider a von Neumann algebra $(\mathcal{M}$, $\|\cdot\|)$ (in general, $\mathcal{M}$ can be a ${ }^{*}$-algebra with unit $\mathbf{1}$ ) which contains a $\mathbf{C}^{*}$-algebra $\mathcal{A}$ with the same unit. We will be interested in (Markov) semigroups $\mathbb{R}^{+} \ni t \mapsto P_{t}: \mathcal{M} \rightarrow \mathcal{M}$ such that: (i) $P_{t}$ is a strongly continuous map for each $t \in \mathbb{R}^{+}$; (ii) $P_{t}(\mathbf{1})=\mathbf{1}$; (iii) $P_{t}\left(f^{*} f\right) \geq 0$ for any $f \in \mathcal{M}$; (iv) $P_{t}$ preserves a given state $\varphi$ on $\mathcal{M}$, i.e. $\varphi\left(P_{t} f\right)=\varphi(f)$ for any $t$ and $f ;(\mathrm{v}) P_{t}$ satisfies a detailed balance condition of the form $\left(f, P_{t} g\right)_{\mathcal{H}_{\varphi}}=\left(P_{t} f, g\right)_{\mathcal{H}_{\varphi}}$ where $\mathcal{H}_{\varphi}$ is a Hilbert space containing $\mathcal{M}$ with inner product defined by $\varphi$ in such a way that $(\mathbf{1}, f)_{\mathcal{H}_{\varphi}}=\varphi(f)$, and finally, (vi) $P_{t}$ possesses the Feller property, i.e. $P_{t}(\mathcal{A}) \subset \mathcal{A}$.
\end{abstract}

1991 Mathematics Subject Classification: Primary 82C10; Secondary 46L57.

Research of A.W.M. supported by the KBN grant 2 PO3B 14409.

R.O. and B.Z. would like to acknowledge the support of EPSRC - GR /K95635.

The paper is in final form and no version of it will be published elsewhere. 
To have quantum counterparts of classical Markov semigroups with interesting ergodic properties we are also interested in quantum Markov semigroups $P_{t}$ with some additional properties: i. exhibiting a return to equilibrium $P_{t} f \rightarrow_{t \rightarrow+\infty} \varphi(f) \mathbf{1}$ for $f \in \mathcal{M}$, and ii. having the hypercontractivity property $\left\|P_{t} f\right\|_{L_{p}(\varphi)} \leq\|f\|_{L_{2}(\varphi)}$ for $p>2$, $t \geq T_{0}$ where $\|\cdot\|_{L_{p}(\varphi)}$ stands for a norm on $\mathcal{M}$ defining the noncommutative $L_{p}$-space while $T_{0}$ is a positive constant depending on $p$.

The paper is organized as follows: in Section 2 we briefly sketch a general strategy for constructing jump type semigroup dynamics, in Section 3 we review some of the standard facts on Quantum Spin Systems. Section 4 deals with Quantum Glauber and Kawasaki types of dynamics, while Section 5 is devoted to the study of existence and ergodicity of translation invariant Markov semigroups. In Section 6 we present a brief discussion of quantum diffusion for spin systems. In the final Section 7 we indicate briefly new results concerning the hypercontractivity in noncommutative $L_{p}$ spaces.

2. General strategy of construction of jump type dynamics. In this section we will be concerned with a general construction of jump-type stochastic dynamics (see [1], [8], [9], [10]), i.e. we want to get a Markov semigroup $P_{t} \equiv e^{t \mathcal{L}}: \mathcal{M} \rightarrow \mathcal{M}, t \in \mathbb{R}^{+}$ where

$$
\mathcal{L} \equiv \sum_{i}\left(\mathbf{E}_{i}-\mathbb{1}\right)
$$

and $\mathbf{E}_{i}$ is a completely positive map on $\mathcal{M}$ preserving $\mathbf{1}$.

To carry out such a construction let us assume that $\mathcal{M}$ is a von Neumann algebra, $\varphi_{1}$ a faithful state on $\mathcal{M}$ (in further applications $\varphi_{1}$ will be taken as a locally normal state). The modular automorphism related to the pair $\left(\mathcal{M}, \varphi_{1}\right)$ will be denoted by $\sigma_{t}^{1}$. Let us define on $\mathcal{M}$ the following inner product (cf. [7], [8], [9]):

$$
<f, g>_{1, s} \stackrel{\text { def }}{=} \varphi_{1}\left(\left(\sigma_{\frac{i s}{2}}^{1}(f)^{*}\left(\sigma_{\frac{i s}{2}}^{1}(g)\right)\right)\right.
$$

where $s \in[0,1]$, and $f, g$ are analytic elements in $\mathcal{M}$ for the modular automorphism $\sigma_{t}^{1}$. The closure of $\mathcal{M}$ with respect to the norm induced by the above inner product leads to a Hilbert space $\mathcal{H}_{1, s}$ associated with $\left(\mathcal{M}, \varphi_{1}\right)$. To simplify notation we will use the convention

$$
<f, g>_{1, s=\frac{1}{2}} \equiv<f, g>_{1}
$$

Let $\mathbf{E}_{0}$ be a conditional expectation, i.e. $\mathbf{E}_{0}\left(f^{*} f\right) \geq 0, \mathbf{E}_{0}(\mathbf{1})=\mathbf{1}, \mathbf{E}_{0}^{2}=\mathbf{E}_{0}$. We define

$$
\varphi_{2}(\cdot) \stackrel{\text { def }}{=} \varphi_{1} \circ \mathbf{E}_{0}(\cdot)
$$

Suppose $\varphi_{2}$ is another faithful state on $\mathcal{M}$. Then, the Takesaki theorem implies that $\mathbf{E}_{0}$ commutes with $\sigma_{t}^{2}$ (the modular automorphism for $\left(\mathcal{M}, \varphi_{2}\right)$ ) and hence is symmetric in $\left(\mathcal{H}_{2, \frac{1}{2}},<\cdot, \cdot>_{2, \frac{1}{2}} \equiv<\cdot, \cdot>_{2}\right)$.

Let $V_{t} \equiv\left(D \varphi_{1}: D \varphi_{2}\right)_{t}$ be the Radon-Nikodym cocycle. We remind that, in particular, $\sigma_{t}^{1}(f)=V_{t}^{*} \sigma_{t}^{2}(f) V_{t}$. The main difficulty in carrying out the presented construction of the Markov generator is the existence of analytic extension of $\mathbb{R} \ni t \mapsto V_{t} \in \mathcal{M}$. The following condition guarantees the desired extension (see [4]): 
Suppose there exists a positive constant $c \in(0, \infty)$ such that for any $0 \leq f \in \mathcal{M}$ the following inequalities hold:

$$
\frac{1}{c} \varphi_{1} \leq \varphi_{2}(f) \leq c \varphi_{1}(f) .
$$

Then, $V_{t}$ extends analytically to $-\frac{1}{2} \leq \operatorname{Im} z \leq \frac{1}{2}$ and $\xi \equiv V_{t \mid t=-\frac{i}{2}}$ is a bounded operator in $\mathcal{M}$. Let us note that the above inequalities also guarantee that $\varphi_{2}$ is a faithful state provided that $\varphi_{1}$ has this property. We can now formulate the main result of this section (cf. [9]):

TheOREM 1. Assume that $\xi \equiv V_{t \mid t=-\frac{i}{2}}$ is a bounded operator in $\mathcal{M}$ and define

$$
\mathbf{E}(f) \stackrel{\text { def }}{=} \mathbf{E}_{0}\left(\xi^{*} f \xi\right) \text {. }
$$

Then, the generalized conditional expectation $\mathbf{E}(\cdot)$ is well defined and it has the following properties:

$$
\mathbf{E}(\mathbf{1})=\mathbf{1}, \quad \mathbf{E}\left(f^{*} f\right) \geq 0, \quad<\mathbf{E}(f), g>_{1}=<f, \mathbf{E}(g)>_{1} .
$$

This theorem ensures that the operator given by:

$$
\mathcal{L} \stackrel{\text { def }}{=} \mathbf{E}-\mathbb{1}
$$

is a well defined Markov generator.

3. Applications to Quantum Spin Systems. This section contains a brief exposition of basic features of quantum systems on a lattice (cf. [3]). We begin with a definition of the basic $\mathbf{C}^{*}$-algebra; a $\mathbf{C}^{*}$-algebra $\mathcal{A}$, with norm $\|\cdot\|$, is defined as the inductive limit over a finite dimensional complex matrix algebra $\mathbf{M}$. By analogy with the classical commutative spin systems it is natural to view $\mathcal{A}$ as a noncommutative analogue of the space of bounded continuous functions. To every finite set $X$ of the lattice $\mathbb{Z}^{d}$, (which is denoted later on by $X \subset \subset \mathbb{Z}^{d}$ ), we associate a subalgebra $\mathcal{A}_{X}$ of operators localized in the set $X$. Let $\mathcal{F}$ denote the family of all finite subsets of $\mathbb{Z}^{d}$. For an arbitrary subset $\Lambda \subset \mathbb{Z}^{d}$ one defines $\mathcal{A}_{\Lambda}$ to be the smallest (closed) subalgebra of $\mathcal{A}$ containing $\bigcup\left\{\mathcal{A}_{X}: X \subset \subset \mathbb{Z}^{d}, X \subset \Lambda\right\}$. An operator $f \in \mathcal{A}$ will be called local if there is some $Y \subset \subset \mathbb{Z}^{d}$ such that $f \in \mathcal{A}_{Y}$. The subset of $\mathcal{A}$ consisting of all local operators will be denoted by $\mathcal{A}_{0}$.

Together with the algebra $\mathcal{A}$ we are given the family $\operatorname{Tr}_{X}, X \subset \subset \mathbb{Z}^{d}$, of normalized partial traces on $\mathcal{A}$. We recall that the partial traces $\operatorname{Tr}_{X}$ have all natural properties of classical conditional expectations. Moreover the family $\left\{\operatorname{Tr}_{X}: X \subset \subset \mathbb{Z}^{d}\right\}$ is compatible in the similar sense as conditional expectations and one can see that there is a unique state $\operatorname{Tr}$ on $\mathcal{A}$, called the normalized trace, such that

$$
\operatorname{Tr}\left(\operatorname{Tr}_{X} f\right)=\operatorname{Tr}(f)
$$

for every $X \subset \subset \mathbb{Z}^{d}$, i.e. the normalized trace can be regarded as a (free) Gibbs state in the similar sense as in the classical statistical mechanics.

A system with an interaction is described using a notion of an interaction potential, i.e. a family $\Phi \equiv\left\{\Phi_{X} \in \mathcal{A}_{X}\right\}_{X \subset \subset \mathbb{Z}^{d}}$ of selfadjoint operators in $\mathcal{A}$. A Banach space of 
potentials satisfying

$$
\|\Phi\|_{n} \equiv \sup _{i \in \mathbb{Z}^{d}} \sum_{\substack{X \subset \subset \mathbb{Z}^{d} \\ X \ni i}}|X|^{n-1}\left\|\Phi_{X}\right\|<\infty
$$

will be denoted by $\mathbb{B}_{n}$. The potentials in $\mathbb{B}_{1}$ will be called Gibbsian. A potential $\Phi \equiv\left\{\Phi_{X}\right\}_{X \subset \subset \mathbb{Z}^{d}}$ is of finite range $R \geq 0$ iff $\Phi_{X}=0$ for all $X \in \mathcal{F}$, $\operatorname{diam}(X)>R$. The corresponding Hamiltonian $H_{\Lambda}$ in $\Lambda \subset \subset \mathbb{Z}^{d}$ is defined by

$$
H_{\Lambda} \stackrel{\text { def }}{=} H_{\Lambda}(\Phi) \equiv \sum_{X \subset \Lambda} \Phi_{X}
$$

Using the Hamiltonian $H_{\Lambda}$ we introduce a density matrix $\rho_{\Lambda} \equiv e^{-\beta H_{\Lambda}} / \operatorname{Tr} e^{-\beta H_{\Lambda}}$ with $\beta \in(0, \infty)$, and define a finite volume Gibbs state $\omega_{\Lambda}$ by

$$
\omega_{\Lambda}(f) \stackrel{\text { def }}{=} \operatorname{Tr}\left(\rho_{\Lambda} f\right)
$$

It is known, see e.g. [3], that for $\beta \in(0, \infty)$ the limit state $\omega \equiv \lim _{\mathcal{F}_{0}} \omega_{\Lambda}$ (defined with some exhaustion $\mathcal{F}_{0}$ of the lattice) exists and is faithful on $\mathcal{A}$. For a quantum spin system, we can also introduce a natural Hamiltonian dynamics defined in a finite volume as the following automorphism group associated to a potential $\Phi$ :

$$
\alpha_{t}^{\Lambda}(f) \equiv e^{+i t H_{\Lambda}} f e^{-i t H_{\Lambda}} .
$$

With this dynamics one has the following KMS condition for the finite volume state $\omega_{\Lambda}$ :

$$
\omega_{\Lambda}\left(f^{*} g\right)=\omega_{\Lambda}\left(\alpha_{-i \beta}^{\Lambda}(g) f^{*}\right)
$$

If $\Phi \in \mathbb{B}_{2}$, then the following limit exists, [13]:

$$
\alpha_{t}(f) \equiv \lim _{\mathcal{F}_{0}} \alpha_{t}^{\Lambda}(f)
$$

for every $f \in \mathcal{A}_{0}$, where $\Lambda \rightarrow \mathbb{Z}^{d}$ through a Fisher sequence $\mathcal{F}_{0}$, where $\mathcal{F}_{0}$ is an increassing sequence of finite volumes invading all the lattice $\mathbb{Z}^{d}$. The generator of this automorphism group $\alpha_{t}$ is given on the local elements by

$$
\delta_{\Phi}(f) \equiv \lim _{\mathcal{F}_{0}} \delta_{\Phi, \Lambda}(f) \equiv \lim _{\mathcal{F}_{0}} i\left[H_{\Lambda}(\Phi), f\right]
$$

where $\left[F_{1}, F_{2}\right] \equiv F_{1} F_{2}-F_{2} F_{1}$ stands for the commutator of two operators $F_{1}$ and $F_{2}$.

The infinite volume state $\omega$ is called an $\left(\alpha_{t}, \beta\right)$-KMS state. By $\mathcal{M}$ we will denote the von Neumann algebra obtained via GNS construction using the state $\omega$, i.e. $\mathcal{M} \equiv($ weak)closure $\left\{\pi_{\omega}(\mathcal{A})\right\}$. We denote by $\varphi_{1}$ the weak extension of $\omega$ on $\mathcal{M}$. $\mathcal{M}$ can be equipped with the following inner product:

$$
<\pi_{\omega}(f), \pi_{\omega}(g)>_{1} \equiv \lim _{\Lambda \uparrow \mathbb{Z}^{d}} \operatorname{Tr}\left\{\left(\varrho_{\Lambda}^{\frac{1}{4}} f \varrho_{\Lambda}^{\frac{1}{4}}\right)^{*}\left(\varrho_{\Lambda}^{\frac{1}{4}} g \varrho_{\Lambda}^{\frac{1}{4}}\right)\right\}
$$

where $\varrho_{\Lambda}$ is the density matrix of the $\left(\alpha_{t}, \beta\right)$-KMS state $\omega$ restricted to $\mathcal{A}_{\Lambda}$ with respect to $\operatorname{Tr}$, i.e. $\omega(\cdot)_{\mid \mathcal{A}_{\Lambda}}=\operatorname{Tr}\left\{\varrho_{\Lambda} \cdot\right\}$. We recall that using Lieb-Epstein concavity results, [5], [6], one can show that for $p \in(2, \infty)$

$$
\left\{\|f\|_{p, \Lambda}\right\}_{\Lambda} \equiv\left\{\left(\operatorname{Tr}\left|\varrho_{\Lambda}^{\frac{1}{2 p}} f \varrho_{\Lambda}^{\frac{1}{2 p}}\right|^{p}\right)^{\frac{1}{p}}\right\}_{\Lambda}
$$

converges for any $f \in \mathcal{A}_{0}$ (as $\Lambda \uparrow \mathbb{Z}^{d}$ ). This leads to the well defined family of norms on $\mathcal{M}$ and the well defined inner product on $\mathcal{M}$. Using them, we can define an interpolating 
family of (quantum) $\mathbb{L}_{p}(\omega), 1 \leq p \leq \infty$ spaces associated to quantum system on a lattice, cf. [7]-[10] and [14]. In particular, for $p=2$ we have the Hilbert space $\mathcal{H}_{\varphi_{1}}$ with the above defined inner product.

4. Quantum Glauber and Kawasaki types dynamics. In this section we indicate how techniques introduced in Sections 2 and 3 may be used in the construction of Markov semigroups. The first model can be considered as a quantum counterpart of generalized Glauber dynamics (see also Section 5, Remarks 3 and 4). Again, let $\mathcal{M}$ denote the von Neumann algebra obtained via GNS construction using the state $\omega$. The partial trace $\operatorname{Tr}_{X}$, for $X \subset \subset \mathbb{Z}^{d}$, can be naturally extended to this von Neumann algebra. Namely

$$
\mathbf{E}_{0, X}(f) \equiv \operatorname{Tr}_{X} f \stackrel{\text { def }}{=} \int d \nu_{X}(U) \pi_{\omega}(U)^{*} f \pi_{\omega}(U)
$$

where $d \nu_{X}$ is the Haar measure on the set of all unitaries in $\mathcal{A}_{X}$. Using it we can introduce the following generalized conditional expectation ([1], [2], [7], [8]):

$$
E_{X}(f) \stackrel{\text { def }}{=} \operatorname{Tr}_{X}\left(\gamma_{X}^{*} f \gamma_{X}\right)
$$

with some bounded operator $\gamma_{X} \in \mathcal{M}$. The next theorem says that this definition is perfectly legitimate for a class of quantum spin systems. Namely, denoting $\varphi_{2} \stackrel{\text { def }}{=} \varphi_{1} \circ \mathbf{E}_{0, X}$ and by $\mathbb{B}_{\text {exp }}$ the following class of potentials:

$$
\|\Phi\|_{e x p} \equiv \sup _{i \in \mathbb{Z}^{d}} \sum_{\substack{X \subset \subset \mathbb{Z}^{d} \\ X \ni i}} e^{\varepsilon|X|}|| \Phi_{X} \|<\infty
$$

for some $\varepsilon>0$, we have (cf. [9])

TheOREm 2. Suppose a system with interaction $\Phi \in \mathbb{B}_{\text {exp }}$ is at high temperatures $|\beta|<\beta_{0}$, with some $\beta_{0}>0$ sufficiently small, or the system is one dimensional, has finite range interaction but its temperature is arbitrary $\beta \in(0, \infty)$. Then, for some positive $c \in(0, \infty)$

$$
\frac{1}{c} \varphi_{1}\left(f^{*} f\right) \leq \varphi_{2}\left(f^{*} f\right) \leq c \varphi_{1}\left(f^{*} f\right) .
$$

Hence, the corresponding Radon-Nikodym cocycles have analytic extension and therefore $\gamma_{X} \stackrel{\text { def }}{=}\left(D \varphi_{1}: D \varphi_{2}\right)_{\mid t=-\frac{i \beta}{2}} \in \mathcal{M}$. Hence

$$
\mathbf{E}_{X}(f) \stackrel{\text { def }}{=} \operatorname{Tr}_{X}\left(\gamma_{X}^{*} f \gamma_{X}\right)
$$

defines a generalized conditional expectation which is symmetric in $\mathcal{H}_{\varphi_{1}}$.

Using the above generalized conditional expectation one can define the following elementary bounded Markov generator:

$$
\mathcal{L}_{X}(f) \equiv E_{X}(f)-f
$$

COROllary. The Markov semigroup given on $\mathcal{M}$ by

$$
P_{t} \stackrel{\text { def }}{=} e^{t \mathcal{L}_{X}}
$$

satisfies

$$
<P_{t} f, g>_{1}=<f, P_{t} g>_{1} \text {. }
$$


This corollary gains in interest if one realizes that $P_{t}$ is a semigroup of selfadjoint contractions (the last property of $P_{t}$ follows from the definition of $\mathcal{L}_{X}$ and the fact that $\mathbf{E}_{X}$ is a selfadjoint contraction). Namely, for any selfadjoint semigroup of contractions one has

$$
\lim _{t \rightarrow+\infty} P_{t}=\mathcal{Q}
$$

where $\mathcal{Q}$ is an orthogonal projection. In other words, such a dynamics manifests a return to equilibrium. Let us add that the state $\varphi_{1}$ is $P_{t}$-invariant:

$$
\varphi_{1}\left(P_{t} f\right)=<P_{t} f, \mathbf{1}>_{1}=<f, P_{t} \mathbf{1}>_{1}=<f, \mathbf{1}>_{1}=\varphi_{1}(f) .
$$

Clearly, it would be desirable to formulate conditions which guarantee the uniqueness of the limit state. This type of ergodicity will be considered in the next section.

To describe the next example of construction let us take as a true conditional expectation the map $\mathbf{E}_{0, X}$

$$
\mathbf{E}_{0, X}(f) \equiv \tau_{X}(f) \stackrel{\text { def }}{=} \frac{1}{2}\left(i d+a_{x}\right)(f)
$$

where the map $a_{X}: \mathcal{M} \rightarrow \mathcal{M}$ is so chosen that: 1. $a_{X}^{2}=i d, 2$. $a_{X}\left(\pi_{\omega}(f)\right)=\pi_{\omega}(f)$ for $f \in \mathcal{A}_{\mathbb{Z}^{d} \backslash X}$, and 3. $\operatorname{Tr}_{X}\left(a_{X}(f)\right)=\operatorname{Tr}_{X}(f)$. Define $\widetilde{\varphi_{2}} \stackrel{\text { def }}{=} \varphi_{1} \circ \mathbf{E}_{0, X}$. To give the appropriate Markov generator for this type dynamics we need (cf. [10]):

THEOREM 3. Suppose a system with interaction $\Phi \in \mathbb{B}_{\text {exp }}$ is at high temperatures $|\beta|<\beta_{0}$, with some $\beta_{0}>0$ sufficiently small, or the system is one dimensional, has finite range interaction but its temperature is arbitrary $\beta \in(0, \infty)$. Then, for some positive $c \in(0, \infty)$

$$
\frac{1}{c} \varphi_{1}\left(f^{*} f\right) \leq \widetilde{\varphi_{2}}\left(f^{*} f\right) \leq c \varphi_{1}\left(f^{*} f\right) .
$$

Hence, the corresponding Radon-Nikodym cocycles have analytic extension and therefore $\eta_{X} \stackrel{\text { def }}{=}\left(D \varphi_{1}: \widetilde{\left.D \varphi_{2}\right)_{\mid t=-\frac{i \beta}{2}}} \in \mathcal{M}\right.$. Hence

$$
\Lambda_{X}(f) \stackrel{\text { def }}{=} \operatorname{Tr}_{X}\left(\eta_{X}^{*} f \eta_{X}\right)
$$

defines a generalized conditional expectation which is symmetric in $\mathcal{H}_{\varphi_{1}}$.

Again, this theorem allows us to define a Markov semigroup on $\mathcal{M}$ by $P_{t} \stackrel{\text { def }}{=} e^{t \mathcal{L}}$, with generator $\mathcal{L} \equiv \Lambda_{X}-\mathbb{1}$, which satisfies

$$
<P_{t} f, g>_{1}=<f, P_{t} g>_{1} .
$$

ExAmple (Quantum Kawasaki dynamics). Put $X \equiv\{i, j\}$ where $i, j \in \mathbb{Z}^{d}$ and set

$$
a_{X} \equiv \iota_{X^{c}} \otimes T_{i j}
$$

where $\iota_{X^{c}}$ is the unit automorphism on $\pi_{\omega}\left(\mathcal{A}_{\mathbb{Z}^{d} \backslash X}\right)$, and $T_{i j}$ is the exchange automorphism on $\pi_{\omega}\left(\mathcal{A}_{X}\right)$ defined by

$$
T_{i j}\left(e_{i}^{l} \otimes e_{j}^{k}\right) \stackrel{\text { def }}{=} e_{i}^{k} \otimes e_{j}^{l},
$$

$e_{i}^{l} \otimes e_{j}^{k}, k, l=1,2, \ldots, \operatorname{dim} M$, is a basis of $\pi_{\omega}\left(\mathcal{A}_{X}\right)$. Obviously, all assumptions for the map $a_{X}$ (so also for our construction) are satisfied. Therefore, we obtain a Markov semigroup which can be considered as a quantum counterpart of generalized Kawasaki dynamics. 
We close this section with the following open problem:

Question. Could the high-temperature condition of Theorems 2 and 3 be omitted?

5. Translation invariant Markov semigroups. Using the elementary Markov generators introduced in the previous section we wish to define

$$
\mathcal{L}^{(X)} \stackrel{\text { def }}{=} \lim _{\Lambda \uparrow \mathbb{Z}^{d}} \mathcal{L}_{\Lambda}^{(X)} \equiv \lim _{\Lambda \uparrow \mathbb{Z}^{d}} \sum_{l \in \Lambda} \mathcal{L}_{X+l}
$$

where $\mathcal{L}_{X+j} \equiv \mathbf{E}_{X+j}-\mathbb{1}$ with $\mathbf{E}_{X+j} f \equiv \mathbf{E}_{0, X+j}\left(\xi_{X+j}^{*} f \xi_{X+j}\right)$ constructed for a system with (finite range) interaction $\Phi$. In other words we wish to get a translation invariant Markov semigroup with interesting ergodic properties. To give an example of conditions implying the existence of the corresponding translation invariant generators we define:

$$
\begin{aligned}
& \partial_{k} \stackrel{\text { def }}{=} \operatorname{Tr}_{\{k\}}-\mathbb{1} . \\
& \delta_{\Psi}(f) \stackrel{\text { def }}{=} \lim _{\Lambda \uparrow \mathbb{Z}^{d}} i\left[H_{\Lambda}(\Psi), f\right], \text { for } \Psi \in \mathbb{B}_{2} . \\
& d(k, X+j) \stackrel{\text { def }}{=} \text { the distance between the site } k \text { and the set } X+j .
\end{aligned}
$$

Then, we have ([11], [12]):

ThEOREM 4. Suppose $\mathcal{L}_{X+j} \equiv \operatorname{Tr}_{X+j}\left(\xi_{X+j}^{*}(\cdot) \xi_{X+j}\right)-\mathbb{1}$ is a Markov generator defined with the (bounded) operators $\xi_{X+j}$ satisfying the following condition:

$$
\left\|\partial_{k} \xi_{X+j}\right\|_{\mathcal{M}} \leq \frac{c}{(d(k, X+j)+1)^{d+\varepsilon}}
$$

for any $k, j \in \mathbb{Z}^{d}$ with some positive constants $\varepsilon$ and $c$. Then, the infinite volume limit

$$
P_{t} f \stackrel{\text { def }}{=} \lim _{\Lambda \uparrow \mathbb{Z}^{d}} e^{t\left(\mathcal{L}_{\Lambda}^{(X)}+\lambda \delta_{\Psi}\right)} f
$$

exists for any $\lambda \in \mathbb{R}$ and any local $f$. Moreover there are positive constants $\lambda_{0}$ and $c_{0}$ such that if $c<c_{0}$ and $|\lambda|<\lambda_{0}$, then the semigroup $P_{t}$ is strongly ergodic in the sense that

$$
\left\|\left|P_{t} f\right|\right\| \leq e^{-m t}|\|f\||
$$

with some $m \in(0, \infty)$ independent of $f$.

If additionally, $P_{t} \pi_{\omega}(\mathcal{A}) \subseteq \pi_{\omega}(\mathcal{A})$ then

$$
\left\|P_{t} f-\varphi_{1}(f) \mathbf{1}\right\| \leq 2 e^{-m t}\|\| f\|\|
$$

for $f \in \pi_{\omega}(\mathcal{A})$. Here the seminorm $\||\cdot|\|$ is defined as follows:

$$
\|f\|\left\|\equiv \sum_{j \in \mathbb{Z}^{d}}\right\| \partial_{j} f \| .
$$

As an application of the above theorem we describe an example of a Markov semigroup with the Feller property. To this end let $\mathcal{A}^{c l} \subset \mathcal{A}$ be the smallest $\mathbf{C}^{*}$-subalgebra containing $\left\{\sigma^{i} \in \mathcal{A}_{\{i\}} \equiv \mathbf{M} ; i \in \mathbb{Z}^{d}\right\}$. In other words, $\mathcal{A}^{c l}$ is the algebra representing the set of classical observables. To describe classical interactions we take a classical potential

$$
\Phi^{c l} \equiv\left\{\Phi_{X} \in \mathcal{A}^{c l}\right\}_{X \subset \subset \mathbb{Z}^{d}}
$$

We note that such an interaction leads to the hamiltonian dynamics (defined in the same way as described in Section 3) which leaves $\mathcal{A}^{c l}$ pointwise invariant, but it is not trivial 
on $\mathcal{A}$. Moreover, using the prescriptions given in Sections 2 and 3 , if $\Phi^{c l} \in \mathbb{B}_{1}$, we can define a Gibbs state on $\mathcal{A}$ and the generalized conditional expectation

$$
\mathbf{E}_{X}(f) \stackrel{\text { def }}{=} \operatorname{Tr}_{X} \gamma_{X}^{*} f \gamma_{X}
$$

with $\gamma_{X} \in \mathcal{A}^{c l}$. Let $\Psi$ be an arbitrary potential of finite range (in general, not a classical one). We set

$$
\mathcal{L}_{\Lambda} \equiv \sum_{i \in \Lambda}\left(\mathbf{E}_{X+i}-\mathbb{1}\right)+\lambda \delta_{\Psi}
$$

where $\lambda \in \mathbb{R}$. Now we are in a position to give the promised example of a Markov semigroup with the Feller property (cf. [11], [12]).

TheOREM 5. The Markov semigroup

$$
P_{t}=\lim _{\Lambda \uparrow \mathbb{Z}^{d}} e^{t L_{\Lambda}} \equiv e^{t\left(\mathcal{L}^{(X)}+\lambda \delta_{\Psi}\right)}
$$

is a well defined dynamical semigroup satisfying

$$
P_{t}(\mathcal{A}) \subseteq \mathcal{A} .
$$

Let us give some remarks on the translation invariant Markov semigroups described in this section:

Remarks. 1. Suppose the semigroup $e^{t \mathcal{L}^{(X)}}$ is ergodic. Then $P_{t} \equiv e^{t\left(\mathcal{L}^{(X)}+\lambda \delta_{\Psi}\right)}$ is also ergodic provided that $|\lambda|<\lambda_{0}$ for some $\lambda_{0} \in(0, \infty)$.

2. Suppose the finite range potential $\Psi$ is equal to the classical one $\Phi$. Then, a Gibbs state corresponding to the potential $\beta \Phi$ is $P_{t}$-invariant.

3. Suppose $\Psi=\Phi$. Then, $P_{t \mid \mathcal{A}^{c l}} \equiv e^{t \mathcal{L}^{(X)}} \mid \mathcal{A}^{c l}$ is the Glauber dynamics.

4. Assume $\Psi=\Phi$. Then, direct calculations show that $\left\{\mathcal{L}_{X+j}, j \in \mathbb{Z}^{d}, X \subset \subset \mathbb{Z}^{d}\right\}$ do not commute with the modular automorphism group associated with the pair $(\mathcal{A}$, Gibbs state defined by $\Phi)$. Therefore, $P_{t}=e^{t\left(\mathcal{L}^{(X)}+\lambda \delta_{\Phi}\right)}$ is a nontrivial extension of Glauber dynamics.

5. Dirichlet forms associated with $\mathcal{L}^{(X)}$ and $\mathcal{L}^{(Y)}$ in the (noncommutative) Hilbert space $\mathcal{H}_{1} \equiv \mathbb{L}_{2}\left(\varphi_{1}, \frac{1}{2}\right)$ are equivalent for any $X, Y \subset \subset \mathbb{Z}^{d}$. Consequently, the strong ergodicity of $e^{t \mathcal{L}^{(X)}}$ implies $\mathbb{L}_{2}$-ergodicity of $e^{t \mathcal{L}^{(Y)}}$ for any $Y \subset \subset \mathbb{Z}^{d}$.

6. Set $\Psi=0$. Then, we get as a special case the Accardi-Matsui semigroup

$$
P_{t} \equiv e^{t \mathcal{L}}
$$

with the generator $\mathcal{L}=\sum_{j \in \mathbb{Z}^{d}} \mathcal{L}_{j}$, where $\mathcal{L}_{j} f \equiv \operatorname{Tr}_{X+j}\left(a_{X+j}^{*} f a_{X+j}\right)-f$, and $a_{X+j} \equiv$ $T_{j} a_{X}, a_{X} \in \mathcal{A}_{Y}, X \subset Y \subset \subset \mathbb{Z}^{d}$ (T $T_{j}$ is the translation automorphism on the lattice).

We close this section with another open problem.

Question. Give an example of quantum interactions $\Phi$ satisfying the assumptions of Theorem 4.

6. Quantum diffusions for spin systems. To describe this type of dynamics let us denote by $\alpha_{t}$ the hamiltonian automorphism on $\mathcal{A}$ which corresponds to a finite range potential $\Phi$. We need to assume that the following condition is true ([8]). 
Definition. We say that the system $\left(\mathcal{A}, \alpha_{t}\right)$ possesses the Asymptotic Abelianness (AA) property iff

$$
\int_{-\infty}^{+\infty}\left\|\delta_{\alpha_{t}(x)}(f)\right\| d t<+\infty
$$

for any $f$ in a dense subalgebra $\tilde{\mathcal{A}}$ in $\mathcal{A}$ and for any $x \in \mathbf{M}_{j} \equiv T_{j}\left(\mathbf{M}_{0}\right)$ where $\mathbf{M}_{0} \subset \mathbf{M}^{\text {s.a. }}$ is a finite subset in the self-adjoint part of the matrix algebra $\mathbf{M}$ associated with a site of the lattice.

We can now formulate a result describing the Dirichlet form of Quaegebeur, Stragier, Verbeure (QSV), [15], Markov generators (cf. [7], [8]).

Theorem 6. Suppose the condition of Asymptotic Abelianness is satisfied. Then, for any temperature $\beta$, any $s \in[0,1]$, and any $x \in \mathbf{M}_{j}, j \in \mathbb{Z}^{d}$, there exists a real kernel $\mathcal{K}_{s}$ ( a positive definite function belonging to $\mathbb{L}_{1}(\mathbb{R}, d r)$ ) such that

$$
\mathcal{E}_{x}(f, g)=\int_{-\infty}^{+\infty} d u d v \mathcal{K}_{s}(u-v)<\delta_{\alpha_{u}(x)}(f), \delta_{\alpha_{v}(x)}(g)>_{\omega_{\beta \Phi}, s}
$$

is a Dirichlet form of a QSV Markov generator in $\mathbb{L}_{2}\left(\omega_{\beta \Phi}, s\right)$ where $\omega_{\beta \Phi}$ is the Gibbs state determined by the potential $\Phi$ and temperature $\beta$.

To state the next result we need to introduce the following condition, (in which we adopt the above notation).

Definition. We say that the system $\left(\mathcal{A}, \alpha_{t}\right)$ possesses the Hyper Asymptotic Abelianness (HAA) property if $\tilde{\mathcal{A}}=\mathcal{A}_{0}$ and for any $f \in \tilde{\mathcal{A}}$ there exists $\epsilon>0$ such that

$$
\left\|\delta_{\alpha_{t}\left(e_{j}\right)}(f)\right\| \leq c(f)(1+|t|)^{-\frac{(d+1+\epsilon)}{2}}
$$

for every $e_{j} \in \mathbf{M}_{j}, t \in \mathbb{R}$ where $c(f)$ is a positive constant depending on $f$.

The existence of translation invariant dynamics of diffusive type is described in (cf. [7], [8]):

THEOREM 7. Suppose the condition of Hyper Asymptotic Abelianness is satisfied. Then

$$
\mathcal{E}(f, g) \equiv \sum_{j \in \mathbb{Z}^{d}} \mathcal{E}_{e_{j}}(f, g)
$$

is a (densely defined) Dirichlet form of a Markov generator $\mathcal{L}$ in $\mathbb{L}_{2}\left(\omega_{\beta \Phi}, s\right)$.

The condition HAA is essential as it allows one to use the finite speed of propagation estimate of Robinson-Lieb to get a dense domain for $\mathcal{E}$. We close this section with another open question:

Question. Give an example of quantum interactions $\Phi$ for which the corresponding hamiltonian dynamics satisfies HAA.

7. Hypercontractivity in noncommutative $\mathbb{L}_{p}$ spaces. Given an interpolating family $\left\{L_{p}(\varphi)\right\}_{p \in[1, \infty)}$ of noncommutative spaces associated to a Gibbs state $\varphi$ one can 
naturally consider stronger notions of contractivity properties of Markov semigroups. In particular one can define the hypercontractive Markov semigroup by the condition

$$
\left\|P_{t} f\right\|_{L_{q}(\varphi)} \leq\|f\|_{L_{p}(\varphi)}
$$

for some $1<p<q<\infty, t \geq T_{p, q}$, with $T_{p, q}$ being a positive constant depending on $p$ and $q$. The usefulness of this property in the theory of Markov semigroups in the commutative case is well known. In particular it provides a very strong tool for proving strong ergodicity of semigroups on infinite dimensional spaces (see e.g. references in [11] and [14]). Because the semigroup $P_{t}$ is uniquely defined by its generator $\mathcal{L}$, it is natural and very useful to express the properties of $P_{t}$ in terms of $\mathcal{L}$. In the classical $L_{p}$ spaces the sufficient and necessary condition for the hypercontractivity is given by the following Logarithmic Sobolev (LS) inequality:

$$
\int f^{2} \ln |f| d \mu \leq c<f,(-\mathcal{L}) f>+\|f\|_{2}^{2} \ln \|f\|_{2} .
$$

In [14] we have begun to study the corresponding infinitesimal description of hypercontractivity; here we restrict ourselves to brief remarks concerning this direction (for a more detailed account on this subject see [14]). Using the local structure of $L_{p}(\varphi)$ we define a curve passing through positive cones $L_{p}^{+}(\varphi)$ by:

$$
X_{q}^{f}(s)=I_{q+s, q}(f), \quad f \in L_{q}^{+}(\varphi), \quad s \geq 0 .
$$

Here $I_{q, p}$ denotes the map from $L_{p}^{+}(\varphi)$ to $L_{q}^{+}(\varphi)$, which in local spaces $L_{p}\left(\varphi_{\Lambda}\right)$ is much less trivial than in the commutative case and is given explicitly by:

$$
I_{q, p}\left(f_{\Lambda}\right)=\rho_{\Lambda}^{-1 / 2 q}\left(\rho_{\Lambda}^{1 / 2 p} f_{\Lambda} \rho_{\Lambda}^{1 / 2 p}\right)^{p / q} \rho_{\Lambda}^{-1 / 2 q} .
$$

Because $L_{q+s}^{+} \subset L_{q}^{+}$, we can consider $s \rightarrow X_{q}^{f}(s)$ as a curve in one Banach space $L_{q}(\varphi)$ and define

$$
T_{q}(f)=-\left.q \frac{d}{d s} X_{q}^{f}(s)\right|_{s=0},
$$

if the corresponding derivative exists. Locally we get

$$
T_{q}\left(f_{\Lambda}\right)=f_{\Lambda} \rho_{\Lambda}^{1 / 2 q}\left(\ln \rho_{\Lambda}^{1 / 2 q} f_{\Lambda} \rho_{\Lambda}^{1 / 2 q}\right) \rho_{\Lambda}^{-1 / 2 q}-1 / 2 q\left(f_{\Lambda} \ln \rho_{\Lambda}+\left(\ln \rho_{\Lambda}\right) f_{\Lambda}\right)
$$

Hence the noncommutative analogue of the classical $\operatorname{LS}(c, d)$ inequality takes the following form for $f \in L_{2}^{+}(\varphi)$ :

$$
<f, T_{2}(f)>-\|f\|_{2}^{2} \ln \|f\|_{2} \leq c<f,-\mathcal{L} f>+d\|f\|_{2}^{2} .
$$

It is worth pointing out that in the noncommutative case this inequality is a'priori a weaker condition than the hypercontractivity of the semigroup $P_{t}=e^{t \mathcal{L}}$. This is because one needs extra $L_{p}$ regularity of the corresponding Dirichlet form which again is much less trivial than in the classical case. Nevertheless this inequality already implies useful properties of the generator $\mathcal{L}$; for example $\operatorname{LS}(c, d=0)$ inequality gives the spectral gap property for $\mathcal{L}$.

Question. Give an example of quantum interactions $\Phi$ for which the corresponding Markov semigroup is hypercontractive. 


\section{References}

[1] L. Accardi, Topics in Quantum Probability, Phys. Rep. 77 (1981), 169-192.

[2] L. Accardi and C. Cecchini, Conditional Expectations in von Neumann Algebras and a Theorem of Takesaki, J. Func. Anal. 45 (1982), 245-273.

[3] O. Bratteli and D.W. Robinson, Operator Algebras and Quantum Statistical Mechanics, Springer Verlag, New York-Heidelberg-Berlin, vol. I (1979), vol. II (1981).

[4] A. Connes, Sur le Théorème de Radon-Nikodym pour les Poids Normaux Fidèles Semifinis, Bull. Sc. math., $2^{e}$ série, 97 (1973), 253-258.

[5] H. Epstein, Remarks on Two Theorems of E. Lieb, Commun. Math. Phys. 31 (1973), $317-325$.

[6] E. H. Lieb, Convex Trace Functions and the Wigner-Yanase-Dyson Conjecture, Adv. in Math. 11 (1973), 267-288.

[7] A. W. Majewski and B. Zegarliński, On Quantum Stochastic Dynamics and Noncommutative $\mathbb{I}_{p}$ Spaces, Lett. Math. Phys. 36 (1995), 337-349.

[8] A.W. Majewski and B. Zegarliński, Quantum Stochastic Dynamics I: Spin Systems on a Lattice, Math. Phys. Electronic J. 1 (1995), Paper 2.

[9] A.W. Majewski and B. Zegarliński, Quantum Stochastic Dynamics II, Rev. Math. Phys. 8 (1996), 689-713.

[10] A.W. Majewski and B. Zegarliński, On quantum stochastic dynamics, Markov Proc. and Rel. Fields 2 (1996), 87-116.

[11] A.W. Majewski, R. Olkiewicz and B. Zegarliński, Dissipative dynamics for quantum spin systems on a lattice, in: Frontiers in Quantum Physics, Eds. S. C. Lim, R. AbdShukor, K. H. Kwek, Springer Verlag, 1998, 112-126.

[12] A.W. Majewski, R. Olkiewicz and B. Zegarliński, Construction and ergodicity of dissipative dynamics for quantum spin systems on a lattice, J. Phys. A: Math. Gen. 31 (1998), 2045-2056.

[13] T. Matsui, Markov semigroups which describe the time evolution of some higher spin quantum models, J. Func. Anal. 116 (1993), 179-198.

[14] R. Olkiewicz and B. Zegarliński, Hypercontractive Markov Semigroups in Noncommutative $L_{p}$ Spaces, Preprint 1997.

[15] G. Stragier, J. Quaegebeur and A. Verbeure, Quantum detailed balance, Ann. Inst. Henri Poincaré 41 (1984), 25-36. 